\title{
Assessing Pre-service Science Teachers Conception of Geometric Optics (Transmission of Light) in the Selected Special Science Colleges of Education in Ghana.
}

\author{
Humphrey Darkeh Assem, Charles Agyei Amoah \\ Wesley College of Education, Science Department, Kumasi-Ghana \\ OLA College of Education, Science Department, Cape Coast-Ghana
}

\begin{abstract}
The purpose of the study was to find out pre-service science teachers conception about geometric optics in the selected Special Science Colleges of Education in Ghana. The study used a fixed non-experimental descriptive survey that went beyond the descriptive to the interpretive in order to provide explanations of patterns and relationships of the results obtained. Five (5) special Science and Mathematics Colleges of Education from five regions in Ghana were selected for the study. Each class was made up of twenty (20) students totalling one hundred (100) students in all. Questionnaire was used to collect data for the study. It was found out that most pre-service science teachers do not understand the practicalities (reflection and refraction) of the aspect of geometrical optics in the physics syllabus they use in teaching. It is recommended that pre-service science teachers should be taken through the practicalities of geometrical optics in the physics syllabus they use in teaching.
\end{abstract}

Keywords: Conception, Misconception, Geometrical optics

\section{Introduction}

To teach all students according to today's standards, teachers need to have deeper understanding of the subject matter (concepts) so that they can help students create useful cognitive maps, relate one idea to another, and address misconceptions. Teachers need to see how ideas connect across fields and to everyday life. This kind of understanding provides a foundation for pedagogical content knowledge that enables teachers to make ideas accessible to others (Shulman, 1987).

There have been relatively rare studies done to investigate the students' misconceptions in geometrical optics. Misconception studies in geometrical optics show that students have difficulties in understanding vision and the nature and propagation of light. In a study by Bendall, Goldberg, and Galili (1993), they investigated prospective elementary teachers' prior knowledge about light and shadow. They interviewed thirty (30) prospective teachers who were all in their junior or senior year out of which very few had taken a physics course in high school. They found that about $20 \%$ of their subjects tended to explain the shadow phenomenon in terms of a reified shadow (attributing the shadow to the presence of something, rather than to the absence of light). The students also could not explain what will become of the shadow when two light sources were used at the same time. Most of the students reasoned that in the region of geometrical overlap there would be either lightness (full illumination) or darkness (shadow). They did not consider semi darkness. In the study, students had a static general illumination conceptualization in which light only exists in space. For example, students could not explain the brightness of a screen. They did not recognize the role of the light in that process (light had to go from the bulb to the screen). For example, in the interview studies, a student recognized that light must be present to observe mirror images, but did not recognize any explicit role for light in that process. In the interviews most of the students thought that presence of the light was necessary to see the non luminous objects in which they gave to the light a static role. Even if, the students said that for seeing luminous objects light must enter to eyes, they did not draw ray diagrams for this situation. Students also had difficulties in understanding of the idea that light from each 
point on a source goes out in all directions. They thought of light as emanating in only one direction from each source, like flash light beams. In their ray diagrams, they tended to show only single lines going outward from individual points on the bulb which is the root of many students' difficulties in understanding image formation.

Feher and Rice (1988) investigated the middle school children's conceptions of shadow formation. They interviewed 40 children using a protocol that was developed through more than fifty interviews. The children explained the shadow as the presence of something that is pushed, moved or thrown to the screen i.e. as a reified shadow. They gave a material characteristic to the shadow. In their diagrams, there were movements of dark areas or shadows between object and screen. Most of the children gave a role to the light in the shadow formation as initiating the shadow by hitting to the object and pushing it to the screen. Some of these students thought that light reflects off the object and due to this reflection shadow is formed and light carries it to the screen. Moreover, in the study children were asked "Is there a shadow in the dark, where there is no light?" The students thought shadow exists in the dark but they cannot see it. They explained this situation in two different ways. One is that either the object produces the shadow hiding within the object and cannot be produced or cast until the light hits the object and provokes it to do so. The other one is that their visual mechanisms are not operative in the darkness. The researchers also found most of the children had an idea that shadow belongs only to the non-luminous object and it always looks like the object. The students did not consider the role of the light source in the shadow formation. Misconceptions of the students about nature and propagation of light and shadows point out that, students have difficulties in explaining and interpretation of image formation by mirrors and lenses. This has necessitated the need to assess pre-service science teachers' conception of geometric optics (transmission of light) in the selected special science colleges of education in Ghana.

\section{Statement of the Problem}

Many pre-service science shows misconception in geometrical optics. Many students have difficulties in understanding vision and the nature and propagation of light. Langley, Ronen, and Eylon (1997) investigated pre-instruction students' conceptions and representations of optical systems, light propagation, illumination patterns and visual patterns. They found that students did not indicate light emanating from the light source in even single diagram. They showed something existing around the light source, without an explicit connection with it. Light was not shown emanating from the specific points of the light source. Moreover, the path of emanation and propagation of the light was influenced by barriers around the source or by remote optical components. Fetherstonhaugh and Treagust (1992) investigated the 8-10 grades students' (age 13-15 years) understanding of light and its properties and their results showed varied degree of misconceptions. This has necessitated the need to assess the pre-service science teachers' conception of geometric optics (transmission of light).

\section{Purpose of the Study}

The purpose of the study was to find out pre-service science teachers conception about geometric optics in the selected Special Science Colleges of Education in Ghana.

\section{Research Question}

1. What is the extent of pre-service science teachers understanding of geometrical optics?

\section{Methodology}

The study used a fixed non-experimental descriptive survey that went beyond the descriptive to the interpretive in order to provide explanations of patterns and relationships of the results obtained.

The target population consisted of all the final year teacher trainees in the Special Science and Mathematics Colleges of Education in Ghana in the year 2010 totalling seven hundred and fifty (750). Although the study was geared towards all the Special Science and Mathematics Colleges of Education in Ghana of which they are fifteen (15) in the country, it was not practically possible to cover the entire country due to a number of constraints, such as, logistics, time, accessibility and human resources. Since all the colleges in question have practically the same resources, that is, both material and human resources (justifiable in view of the fact that all the science colleges have science laboratories well stock with apparatus and also qualified physics tutors per personal investigation), there was no problem in choosing five (5) colleges from which samples were drawn. 
Five (5) special Science and Mathematics Colleges of Education from five regions in Ghana were selected for the study at based on proximity and accessibility to the colleges in terms of easy transportation. Each class was made up of twenty (20) students totalling one hundred (100) students in all. Questionnaire was used to collect data for the study.

\section{Results/Discussion}

Pre-service science teacher's conceptual understanding of geometric optics is presented on Table 1.

Table 1: Pre-service science teacher's conceptual understanding of geometric optics

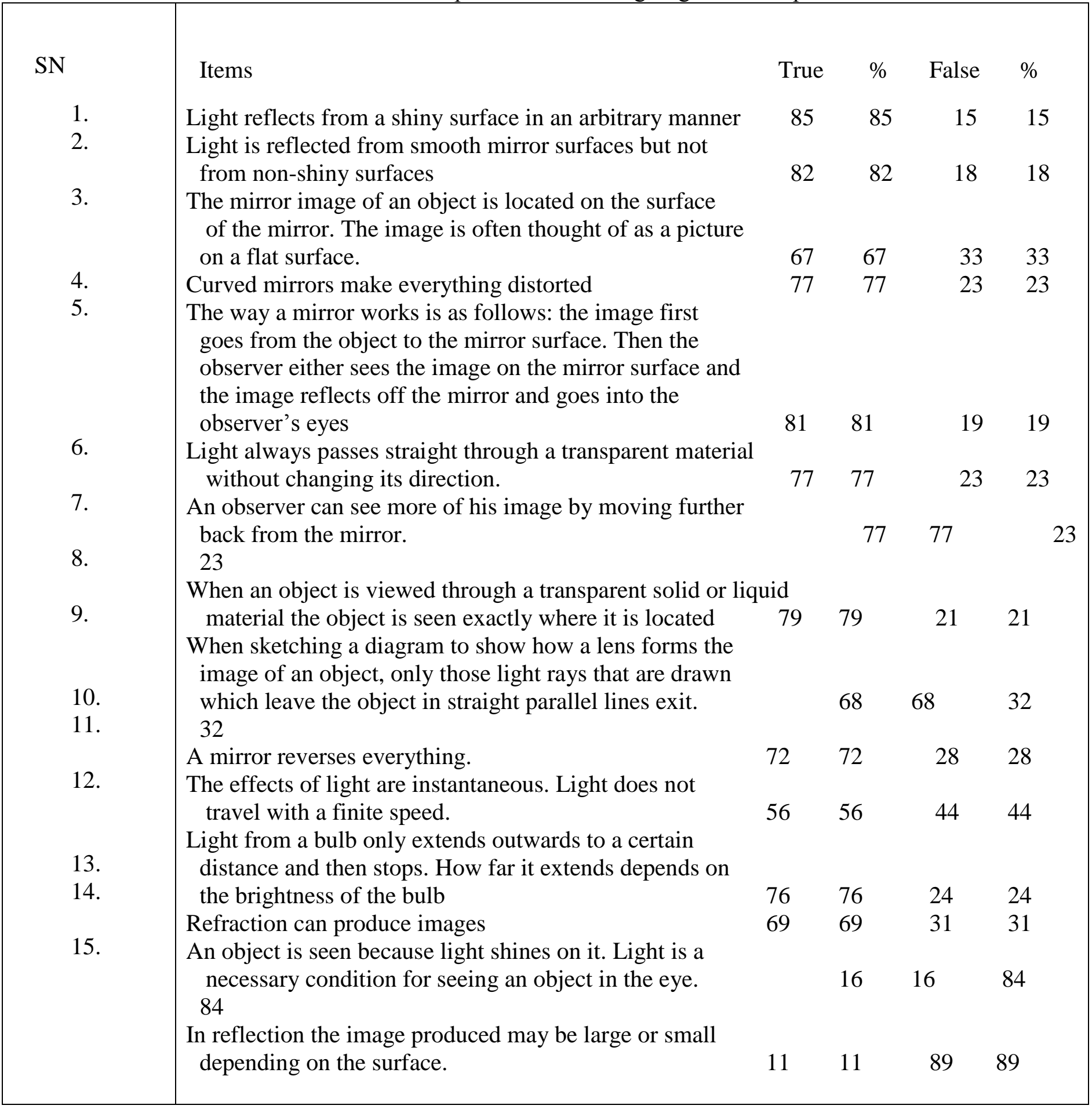

\section{Source: Field data}

From Table 1, eighty five (85) students representing $85 \%$ chose the true option indicating that they did not understand the concept under investigation in questionnaire item one (1) which suggests that light reflects from a shiny surface in an arbitrary manner. The most popular reason they gave was that once light hits any surface it scatters back into the medium from which it came. On the same concept, $16 \%$ of the 
trainees displayed their sound knowledge by choosing false as their option. The most popular of their reasons was that the two types of reflection (regular and diffuse) obey the laws of reflection. This is an indication that they do understand the concept of reflection.

On questionnaire item two (2), eighty two (82) trainees representing $82 \%$ were of the view that the item was true. The most popular of their reasons is that only shinny surfaces reflect light. On the other hand $18 \%$ of the trainees believe that the statement was wrong hence chose false. Their reason was that there are two types of reflection, diffuse reflection (occurs on rough non-shinny surfaces) and regular reflection (occurs on shinny surfaces). It can be concluded that the latter group did understand the concept.

Questionnaire item three (3) recorded sixty seven percent (67\%) false and twenty three percent $(33 \%)$ true. Most of the trainees who chose true were of the view that the mirror is a flat body and hence images can only be formed on the surface. This is obviously a misunderstanding of the concept. Those who chose the true as their option gave their reason as 'images in the mirrors are far behind them as the objects that form them are in front of the mirror.

On item four (4), seventy seven (77) trainees were of the view that the statement 'curve mirrors distort images' is true. Most of them believe that all curved mirrors are the same. This is absolutely a misunderstanding. Moreover, twenty three (23) trainees representing thirty three percent (33\%) believe that there are at least three types of curved mirrors. They named these mirrors as concave, convex and parabolic mirrors hence chose false as their answer.

It was also recorded that eighty one (81) trainees representing $81 \%$ believe that questionnaire item five is true. They believe that the image first goes from the object to the mirror surface and this allows the observer to see the image on the mirror surface. Hitherto, $19 \%$ of the trainees were of the view that images are seen in mirrors because light travels from the object to the mirror and thence reflects into the eye. This is an indication that the later group understands the concept.

Item six (6) recorded seventy seven (77) true(s) as against twenty three (23) false (s). Those who subscribed to the option, 'true' among their reasons was that transparent materials do not obstruct the path of light. However, the opposing group believes that once light travels from one medium into another medium its path will change. Hence they believe the statement is false.

Questionnaire item seven (7), registered seventy seven (77) true and twenty three (23) false. Amongst the popular reasons auctioned by the earlier group was that the more a person moves away from a mirror the bigger they become. Nevertheless, those who chose false indicated that image size in a plane mirror is the same as object size but may vary in curved mirrors. This is evidence that they do understand the concept under investigation.

Seventy nine percent (79\%) of the trainees opted for true on questionnaire item number eight (8), assigning a reason that the object will always be at where it is placed and hence its position will not change. Twenty one (21) trainees however displayed their understanding of the concept by opting for false while citing their reason as 'When an object is viewed through a transparent solid or liquid material the object is seen at different location due to refraction.

On questionnaire item number nine (9), sixty eight (68) of the trainees representing $68 \%$ chose true. They believe that when sketching a diagram to show how a lens forms the image of an object, only those light rays that are drawn which leave the object in straight parallel lines exit because light travels in a straight line. Twenty two percent (22\%) of them believe that there are many other rays except that few rays are required to locate the image of an object based on some principles. This was the basis upon which the chose the option, false.

Recording responses on item number ten (10), it was realized that seventy two (72) of the trainees representing $72 \%$ were of the view that mirrors reverse everything. Meanwhile, $28 \%$ of the trainees did not believe this was the case hence opted for false. Interestingly enough both groups did not give any tangible reason for their choices.

Questionnaire item eleven (11), recorded fifty six percent (56\%) true and forty four percent (44\%) false. The item sought to find out if the effects of light are instantaneous and whether light does not travel with a finite speed. Those who selected true, have among other reasons that light does not travel with a finite speed because the speed of light changes as it moves further away. However their opponents believe that light travels with a finite speed but the speed of light in a medium depends on the refractive index of the medium. 
Furthermore, seventy six (76) of the trainees were of the view that item 12, was true while twenty four (24) objected to their option hence chose false. According to the last group, light from a bulb only extends outwards to a certain distance and then stops. How far it extends depends on the brightness of the bulb because bright bulbs give more light. This is obviously a misunderstanding.

Questionnaire item number thirteen (13) recorded 69 true with a most common reason that we can see our faces in water when light moves from air into water. Thirty one (31) of the trainees opted for false on the basis that it is only reflection that produce image. They argue that the image we see in water is not due to refraction but reflection.

Item 14 sought to investigate trainees understanding of the effect of light on seeing. Sixteen (16) trainees opted for true explaining that light travels from an object into the eye for one to see it. However eighty four (84) of the trainees did not agree with them they however said objects can be seen whether there is light on the object or not. This displays their misunderstanding of the concept under discussion.

Item fifteen (15), received $11 \%$ and $89 \%$ true and false responses respectively. The bone of contention was 'in reflection the image produced may be large or small depending on the surface'. The former group cited among other reasons that nature and size of image depends on the reflecting surface and also the position of the object in front of the reflecting surface. While the latter group agrees that the image of an object is always the same irrespective of the reflecting surface. This is a misunderstanding. Table $1 \mathrm{~b}$, shows the summary of trainees responses to the questionnaire item. It sought to highlight trainees' understanding on the various items.

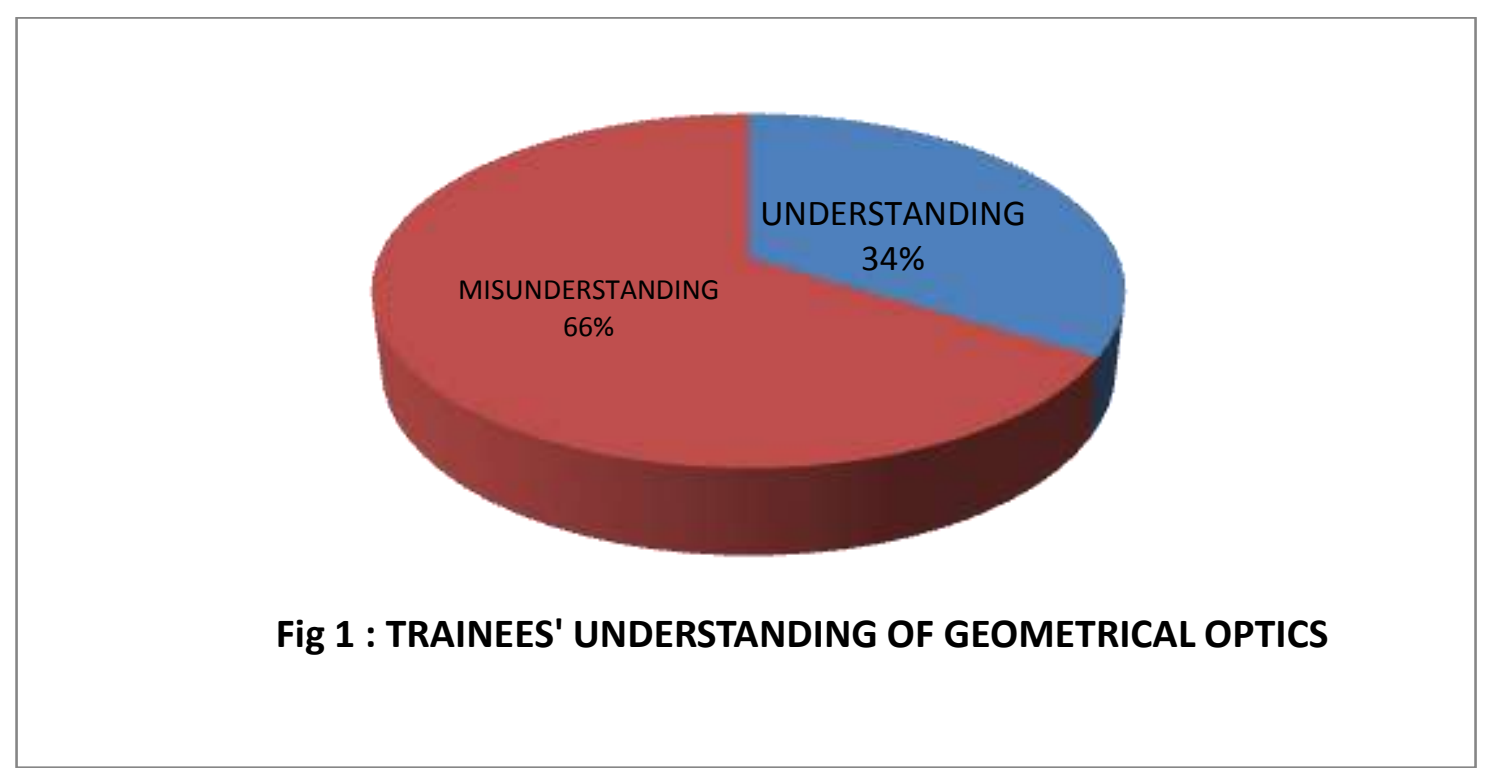

From Fig. 1 , it is evident that $66 \%$ of the trainees do not understand the questionnaire items. This means that only $34 \%$ of the trainees do understand the questionnaire items.

\section{Conclusion}

The purpose of the study was to find out pre-service science teachers conception about geometric optics in the selected Special Science Colleges of Education in Ghana.

It was concluded that most pre-service science teachers do not understand the practicalities (reflection and refraction) of the aspect of geometrical optics in the physics syllabus they use in teaching.

\section{Recommendation}

1. Pre-service science teachers should be taken through the practicalities of geometrical optics in the physics syllabus they use in teaching.

\section{References}

[1] Bendall, S., Goldberg, F., \&Galili, I. (1993). Prospective Elementary Teachers' Prior Knowledge about Light. Journal of Research in Science Teaching, 30 (9), 1169-87. 
[2] Feher, E. \& Rice, K. (1988). Shadows and Anti-Images : Children's Conceptions of Light and Vision. 2. Science Education, 72 (5), 637-649.

[3] Fetherstonhaugh, T., \&Treagust, D. F. (1992). Students' understanding of light and its properties: teaching to engender conceptual change. Science education, 76, 653-672.

[4] Langley, D., Ronen, M., \&Eylon, B. (1997). Light Propagation and Visual Patterns: Pre-instruction Learners' Conceptions. Journal of Research in Science Teaching, 34 (4),399424 